\title{
Diameter-Controllable Magnetic Properties of Co Nanowire Arrays by Pulsed Electrodeposition
}

\author{
Youwen Yang, ${ }^{1}$ Yanbiao Chen, ${ }^{1}$ Yucheng Wu, ${ }^{2}$ Xiangying Chen, ${ }^{1}$ and Mingguang Kong ${ }^{3}$ \\ ${ }^{1}$ School of Chemical Engineering, Hefei University of Technology, Hefei 230009, China \\ ${ }^{2}$ School of Material Science and Engineering, Hefei University of Technology, Hefei 230009, China \\ ${ }^{3}$ Key Laboratory of Material Physics, Institute of Solid State Physics, Chinese Academy of Science, Hefei 230031, China
}

Correspondence should be addressed to Youwen Yang, hfutyyw@163.com

Received 24 November 2009; Revised 16 January 2010; Accepted 20 January 2010

Academic Editor: Yanqiu Zhu

Copyright (C) 2010 Youwen Yang et al. This is an open access article distributed under the Creative Commons Attribution License, which permits unrestricted use, distribution, and reproduction in any medium, provided the original work is properly cited.

\begin{abstract}
The Co nanowires with different diameters were prepared by pulsed electrodeposition into anodic alumina membranes oxide templates. The micrographs and crystal structures of nanowires were studied by FE-SEM, TEM, and XRD. Due to their cylindrical shape, the nanowires exhibit perpendicular anisotropy. The coercivity and loop squareness (Mr/Ms) of Co nanowires depend strongly on the diameter. Both coercivity and $\mathrm{Mr} / \mathrm{Ms}$ decrease with increasing wire diameter. The behavior of the nanowires is explained briefly in terms of localized magnetization reversal.
\end{abstract}

\section{Introduction}

Magnetic nanowires have attracted much interest in the recent years for their potential applications in high-density magnetic recording devices. To realize these applications, the structure and the growth direction of nanowires need to be controlled precisely because the magnetic properties are related to these [1-3]. The synthesis and control of the magnetic nanostructure on a large scale is a challenging issue in the field of material science. One of the solutions is the method to combine electrodeposition with template [4]. Template-assisted electrodeposition is usually advantageous over other "dry" coating technologies due to its low cost, simple equipment, and large-scale processing [5]. Magnetic studies involving template synthesized ferromagnetic nanowire arrays are mainly focused on lowdensity arrays, even single ferromagnetic nanowire arrays of transition metals [6]. As for high density arrays, usually the magnetic behavior of very large aspect ratio wires is comparatively investigated between different metals [7, $8]$ and crystal structures [9, 10]. Therefore, more studies involving densely packed arrays are needed to understand the effect of aspect ratio, packing density on array magnetic properties, and to improve their potential in data storage applications.
As we know, cobalt is a hard magnetic material with tailorable magnetic properties through variation of the structural parameters. It was demonstrated in hcp Co nanowires that depending on texture, the shape and magnetocrystal anisotropies can either compete or add up to yield a partially controllable effective anisotropy $[11,12]$. Highly ordered Co nanowire arrays with uniform diameter are essential for studying their properties and application in the highdensity magnetic recording devices in future. Consequently, it has recently become a popular trend to try to control the crystal structure and magnetic properties of Co nanowire arrays by modifying the deposition parameters $[13,14]$. In this study, the different diameters hcp Co nanowire arrays were fabricated by pulsed electrodeposition into anodic alumina membranes (AAMs). The magnetic properties of Co nanowires have also been investigated.

\section{Experimental}

The AAMs were prepared by a two-step anodization process $[15,16]$. The barrier layer was dissolved in $\mathrm{H}_{3} \mathrm{PO}_{4}$ solution at $30^{\circ} \mathrm{C}$ for 30 minutes and the pore diameter of the asprepared AAM is about $50 \mathrm{~nm}$. While the AAM with pore size of $65 \mathrm{~nm}, 90 \mathrm{~nm}$ is obtained by etching in $\mathrm{H}_{3} \mathrm{PO}_{4}$ solution at $30^{\circ} \mathrm{C}$ for 40 minutes and 60 minutes, respectively. 


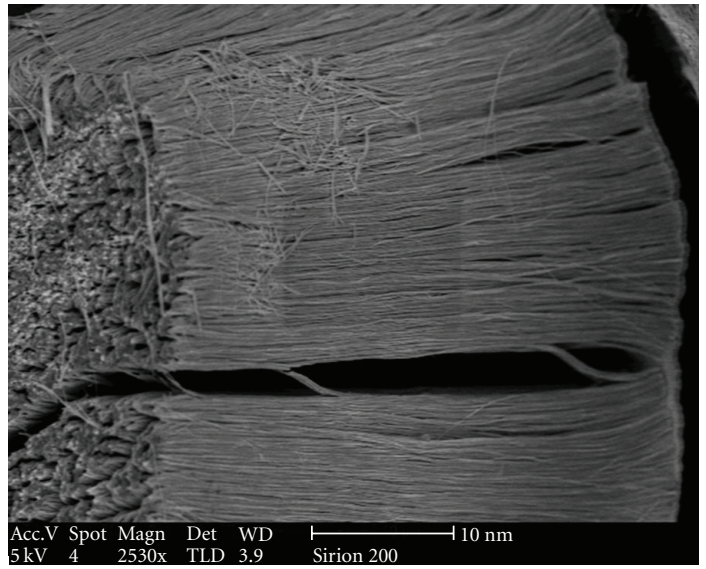

(a)

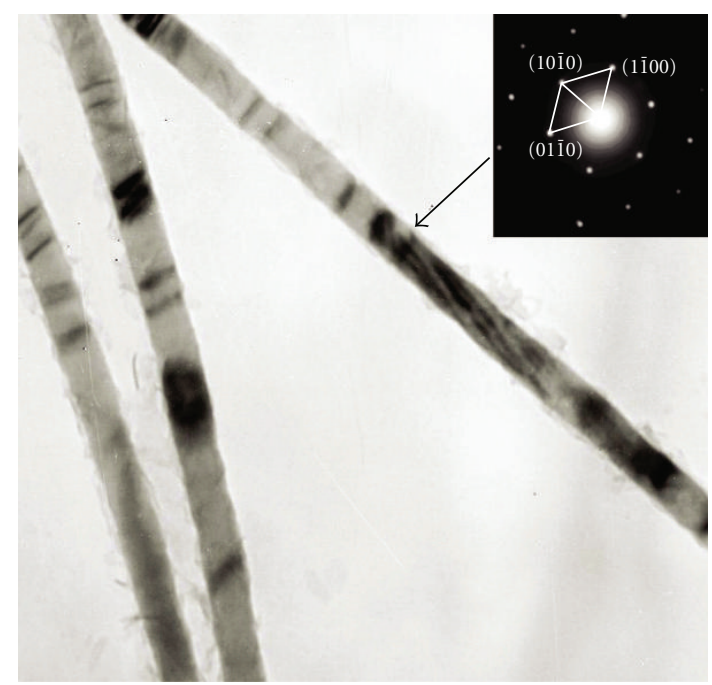

(c)

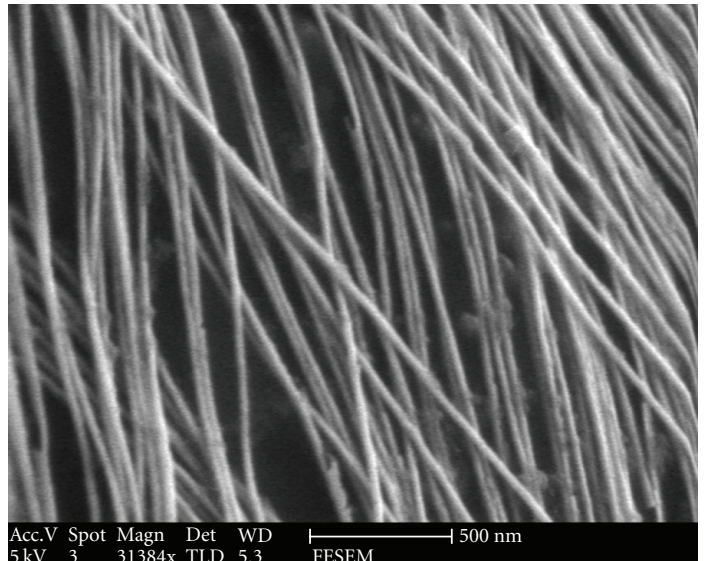

(b)

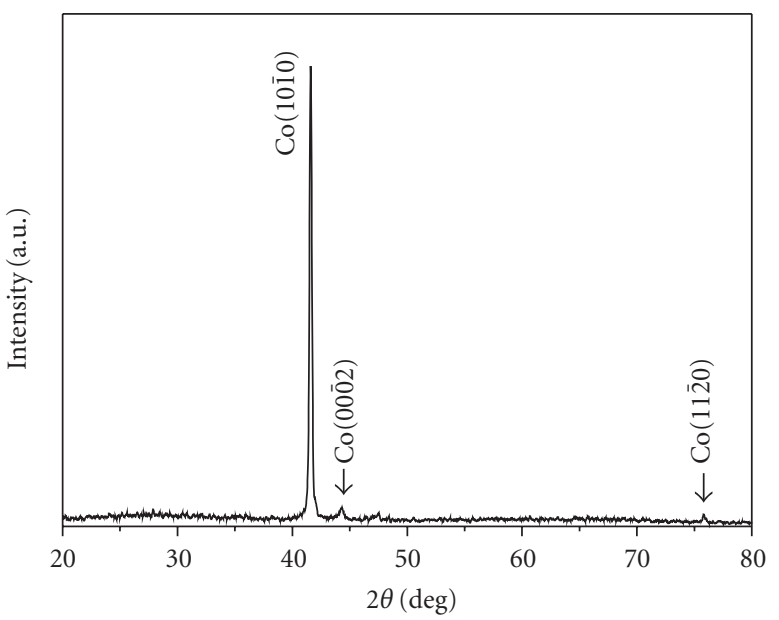

(d)

FIGURE 1: FE-SEM images, TEM images, and XRD pattern of Co nanowires.

Finally a layer of Au film (about $200 \mathrm{~nm}$ ) was sputtered onto one side of AAM to server as the working electrode. The pulsed electrodeposition of the Co nanowire arrays was performed in a two-electrode electrochemical cell controlled by computer, and a graphite plate was used as the counter electrode. The typical electrolyte solution contains $0.2 \mathrm{M}$ $\mathrm{CoSO}_{4} \cdot 7 \mathrm{H}_{2} \mathrm{O}, 40 \mathrm{~g} / \mathrm{L} \mathrm{H}_{3} \mathrm{BO}_{3}$ solution. The $\mathrm{PH}$ value of the electrolyte was adjusted to 5.0 by $1 \mathrm{M} \mathrm{NaOH}$ and the fabrication was performed at $27^{\circ} \mathrm{C}$ at the voltage of $-3.0 \mathrm{~V}$. The pulse frequency was $200 \mathrm{~Hz}\left(T_{\text {on }}=3.75 \mathrm{~ms}, T_{\text {off }}=\right.$ $1.25 \mathrm{~ms}$ ).

The X-ray powder diffraction (XRD) pattern of the nanowire arrays was obtained by an X-ray diffractometer (D/MAX-rA) with $\mathrm{Cu} \mathrm{Ka}$ radiation $(\lambda=1.54178 \AA)$. The morphologies were observed with field emission scanning electron microscope (FE-SEM) performed on JEOL JSM$6700 \mathrm{~F}$ and a transmission electron microscope (TEM) on $\mathrm{H}-$ 800. For the FE-SEM investigation, the sample was immersed in $3 \mathrm{wt} \% \mathrm{NaOH}$ solution to partially remove the alumina template and then washe several times with deionized water.
The TEM specimen was obtained by completely dissolving the AAM in $5 \mathrm{wt} \% \mathrm{NaOH}$ solution and then rinsed with absolute ethanol and immersed in an ultrasonic bath for 8 minutes. A Physical Property Measurement System (PPMS) was used to measure the hysteresis loops of the samples at room temperature.

\section{Results and Discussion}

The Microscopic morphology and XRD pattern of the Co nanowire arrays are shown in Figure 1. A large-area, high density, and high-order Co nanowires were liberated from the AAM and bundled together after partly dissolving the AAM can be seen. The nanowires is long and continuous, the surface is smooth, and diameter is uniform. The length of the nanowires is about $30 \mu \mathrm{m}$ (Figure 1(a)), and the diameter is about $65 \mathrm{~nm}$ (Figures 1(b) and 1(c)). The aspect ratio (length/diameter) of the nanowires is about 460. The XRD pattern of as-prepared Co nanowire arrays is shown in Figure $1(\mathrm{~d})$. All peaks can be indexed to the hcp phase, and 


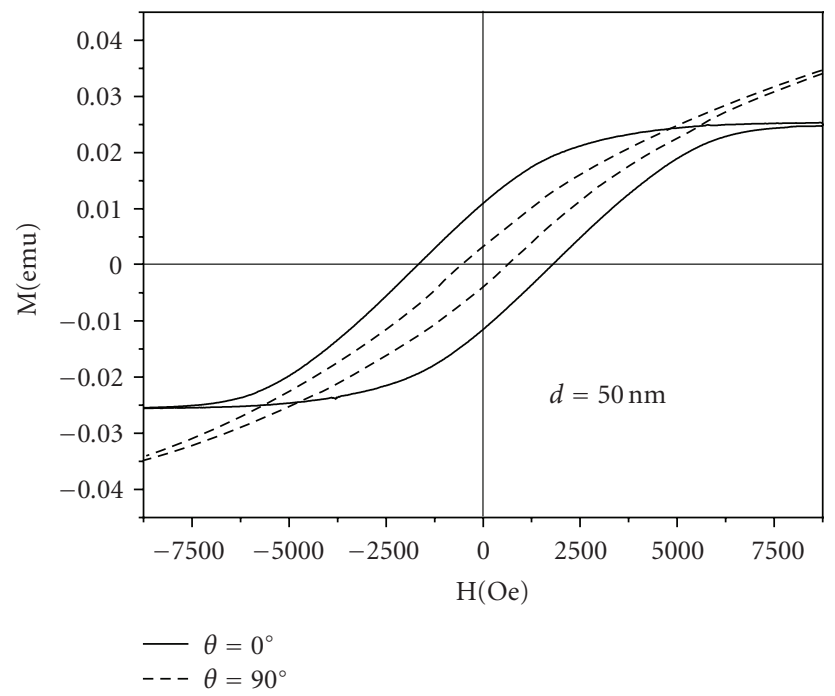

(a)

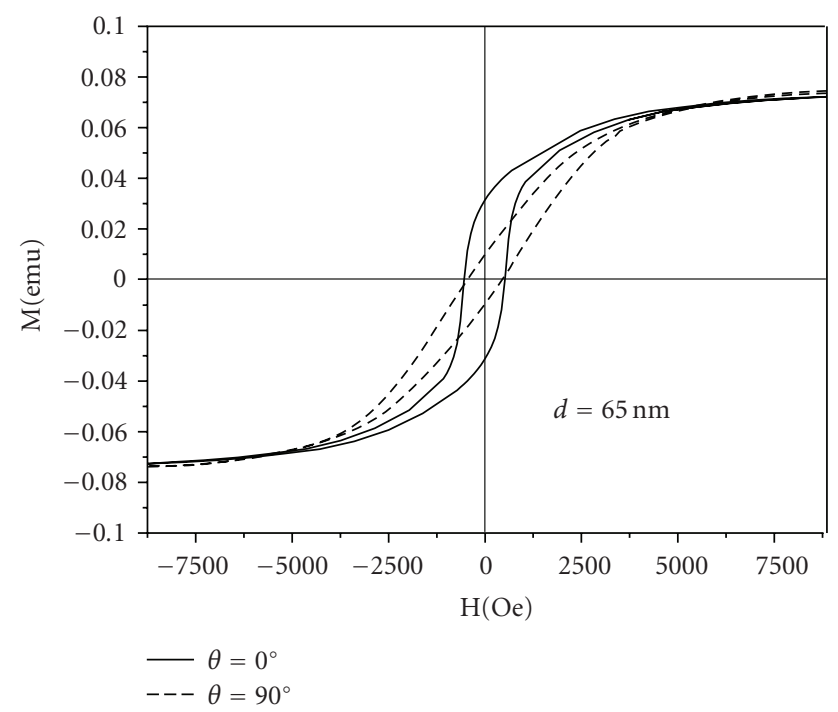

(b)

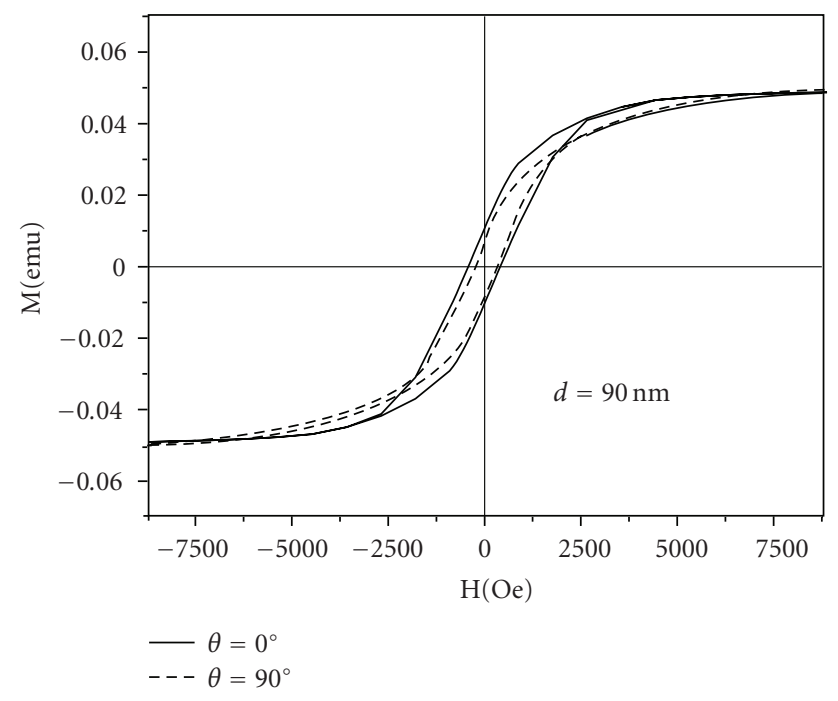

(c)

FIgUre 2: The magnetic hysteresis loops for different diameters Co nanowire arrays of $50 \mathrm{~nm}$ (a), $65 \mathrm{~nm}$ (b), and $90 \mathrm{~nm}$ (c). match well with the standard diffraction peaks of Co (JCPDF 89-4308). The intensity for (10 $\overline{1} 0)$ planes is very strong and higher than others, which indicates that the nanowires have highly preferential orientation. The diffraction pattern which is corresponding to the area signed by the arrow can be indexed to hcp structure, and Co nanowires has a preferred orientation along the $[10 \overline{1} 0]$ direction (Figure $1(\mathrm{c})$ ), which is in consistent with the result of the XRD.

Figure 2 shows the magnetic hysteresis loops for the Co nanowire arrays with different diameter, and the direction of the applied magnetic field is either parallel $\left(\theta=0^{\circ}\right.$, solid lines) or perpendicular $\left(\theta=90^{\circ}\right.$, dashed lines $)$ to the axis of the nanowires. It is worth mentioning that bigger coercivity and loop squareness $(\mathrm{Mr} / \mathrm{Ms})$ ( $\mathrm{Mr}$ is the remanent magnetization) indicate a strong magnetic anisotropy. It can be seen that, as the diameter increases, the Hc $(\theta=$ $\left.0^{\circ}\right) / \mathrm{Hc}\left(\theta=90^{\circ}\right)$ values are $1424 / 584,502 / 396$, and $402 / 349$ Oe; the $\mathrm{Mr} / \mathrm{Ms}\left(\theta=0^{\circ}\right)$ are $50 \%, 36.4 \%, 21.5 \%$, while $\mathrm{Mr} / \mathrm{Ms}\left(\theta=90^{\circ}\right)$ are $20.3 \%, 15.7 \%, 9.8 \%$. The results of the values of coercivity with the diameter of the nanowires illustrate that the shape anisotropy in the magnetization process plays an important role, and the easy axis along the wire (perpendicular to the film plane).

Nanowire magnetization process is generally in three areas by the following factors $[17,18]$ : magnetocrystalline anisotropy, shape anisotropy, and the interaction between the nanowires. Some works $[19,20]$ have reported that the effective anisotropy of the magnetic nanowire arrays comes from the competition between the magnetocrystalline anisotropy and the shape anisotropy. For hcp-phase Co, magnetocrystalline anisotropy energy density $K_{1}=5 \times$ $10^{6} \mathrm{erg} / \mathrm{cm}^{3}$ is comparable to the shape anisotropy energy density $\pi \mathrm{MS}^{2}=6.0 \times 10^{6} \mathrm{erg} / \mathrm{cm}^{3}$ [10], where $\mathrm{M}_{\mathrm{S}}$ is the saturation magnetization of Co. Magnetocrystalline anisotropy of the hcp Co nanowires will make the magnetic moment arrange along the $c$-axis which perpendicular to the nanowire's axis in our experiments, but the shape anisotropy tends to arrange the magnetic moment along the axis of the nanowires [20]. So the competition between magnetocrystalline anisotropy and shape anisotropy results in a relatively weak effective anisotropy along the nanowire's axis, which leads to the low coactivity. When the diameter of the nanowires is small, each magnetic nanowire can be seen as a single domain magnetic structure. As the diameter increases, the single domain structure becomes multidomain. In the magnetization process, single domain structure of nanowires is through the atomic magnetic moment rotation to complete. The energy required to achieve this process than the energy of magnetic domain movement required is much greater. It makes small diameter nanowires with large coercivity. In contrast, the multidomain structure of nanowires is simply by moving magnetic domain to achieve in the magnetization process. This movement of energy consumed is small [21], so large diameter nanowire coercivity is smaller. The $\mathrm{Mr} / \mathrm{Ms}$ changed by varying diameter is also this reason, when the external magnetic field becomes zero, the $\mathrm{Mr} / \mathrm{Ms}$ decreases fast by the interaction of magnetic domains in the multidomain structure nanowires. That is the main reason that coercivity and $\mathrm{Mr} / \mathrm{Ms}$ decrease 
rapidly with increasing wire diameter from $50 \mathrm{~nm}$ to $65 \mathrm{~nm}$.

Moreover, with increasing $d$, in-plane saturation field decreases (with perpendicular loop becoming more square) and out-of-plane saturation field dramatically rises (with parallel loop becoming more sheared). Contrary to squarelike hysteresis loops of isolated (i.e., noninteracting) Co NWs [22] the parallel hysteresis loops are significantly sheared, hinting further on the presence of strong magnetostatic interactions between NWs. Even though the sample with the thickest wires $(d=90 \mathrm{~nm})$ behaves similar to bulklike ferromagnetic thin films, the out-of-plane (in-plane) coercivity still remains large (small). This can be attributed to the wire-like nature of the studied films. As the diameter increases from $65 \mathrm{~nm}$ to $90 \mathrm{~nm}$, the coercivity and $\mathrm{Mr} / \mathrm{Ms}$ decrease slowly. It should primarily be attributed to the evolution of magnetic interactions.

\section{Conclusions}

In summary, densely packed hcp Co nanowire arrays of varying diameter were obtained via plused electrodeposition into the pores of AAM. Dependence of magnetic properties on diameter, that is, aspect ratio, was investigated using PPMS at room temperature. It was observed that the magnetization easy axis lies parallel to Co nanowire axis. At the same time, the magnetic parameters, including coercivity, squareness, are highly correlated with diameter. So we can manipulate the magnetic property of Co nanowire arrays by changing the diameter of the nanowires.

\section{Acknowledgment}

The project was supported by the National Natural Science Foundation of China (10847115).

\section{References}

[1] K. Nielsch, R. B. Wehrspohn, J. Barthel, et al., "Electrochemical control and selection of the structural and magnetic properties of cobalt nanowires," Applied Physics Letter, vol. 79, p. 1360, 2001.

[2] F. Tian, J. Zhu, and D. Wei, "Fabrication and magnetism of radial-easy-magnetized Ni nanowire arrays," Journal of Physical Chemistry C, vol. 111, no. 34, pp. 12669-12672, 2007.

[3] H. P. Liang, Y. G. Guo, J. S. Hu, et al., "Ni-Pt multilayered nanowire arrays with enhanced coercivity and high remanence ratio," Inorganic Chemistry, vol. 44, no. 9, pp. 3013-3015, 2005.

[4] G. W. She, L. X. Mu, and W. S. Shi, "Electrodeposition of onedimensional nanostructures," Recent Patents on Nanotechnology, vol. 3, no. 3, pp. 182-191, 2009.

[5] X. W. Wang, G. T. Fei, P. Tong, X. J. Xu, and L. D. Zhang, "Structural control and magnetic properties of electrodeposited Co nanowires," Journal of Crystal Growth, vol. 300, no. 2, pp. 421-425, 2007.

[6] R. Ferre, K. Ounadjela, J. M. George, L. Piraux, and S. Dubois, "Magnetization processes in nickel and cobalt electrodeposited nanowires," Physical Review B, vol. 56, no. 21, pp. 14066-14075, 1997.
[7] Q. Liu, J. Wang, Z. Yan, and D. Xue, "Characterization and magnetic properties of $\mathrm{Fe}_{1-x} \mathrm{Ni}_{x}$ nanowire arrays," Physical Review B, vol. 72, no. 14, pp. 1-6, 2005.

[8] W. Chen, S. L. Tang, M. Lu, and Y. W. Du, "The magnetic properties and reversal of Fe-Co nanowire arrays," Journal of Physics Condensed Matter, vol. 15, no. 26, pp. 4623-4630, 2003.

[9] J. U. Cho, J. H. Wu, J. H. Min, et al., "Control of magnetic anisotropy of Co nanowires," Journal of Magnetism and Magnetic Materials, vol. 303, no. 2, pp. e281-e285, 2006.

[10] J. Silverberg, A. Friedman, and L. Menon, "Growth and magnetic properties of polycrystalline self-assembled bifurcated co nanowires," Journal of Nanomaterials, vol. 2008, Article ID 782930, 7 pages, 2008.

[11] O. Yalçin, F. Yildiz, M. Özdemir, et al., "Ferromagnetic resonance studies of Co nanowire arrays," Journal of Magnetism and Magnetic Materials, vol. 272-276, no. 3, pp. 1684-1685, 2004.

[12] X. W. Wang, Z. H. Yuan, S. Q. Sun, et al., "Thermal expansion behaviors of hcp and fcc Co nanowire arrays," Physics Letters A, vol. 373, no. 32, pp. 2887-2889, 2009.

[13] J. U. Cho, J. H. Min, S. P. Ko, et al., "Effect of external magnetic field on anisotropy of $\mathrm{Co} / \mathrm{Cu}$ multilayer nanowires," Journal of Applied Physics, vol. 99, no. 8, p. 08C909, 2006.

[14] J. H. Min, J. U. Cho, Y. K. Kim, et al., "Substrate effects on microstructure and magnetic properties of electrodeposited Co nanowire arrays," Journal of Applied Physics, vol. 99, no. 8, Article ID 08Q510, 2006.

[15] Y. W. Yang, L. Li, X. H. Huang, G. H. Li, and L. D. Zhang, "Fabrication and optical property of single-crystalline InSb nanowire arrays," Journal of Materials Science, vol. 42, no. 8, pp. 2753-2757, 2007.

[16] L. Li, Y. W. Yang, X. H. Huang, G. H. Li, and L. D. Zhang, "Pulsed electrodeposition of single-crystalline $\mathrm{Bi}_{2} \mathrm{Te}_{3}$ nanowire arrays," Nanotechnology, vol. 17, no. 6, pp. 17061712, 2006.

[17] H. N. Hu, H. Y. Chen, S. Y. Yu, et al., “Textured Co nanowire arrays with controlled magnetization direction," Journal of Magnetism and Magnetic Materials, vol. 295, no. 3, pp. 257262, 2005.

[18] M. Vazquez, K. Pirota, J. Torrejon, D. Navas, and M. Hernandez-Velez, "Magnetic behaviour of densely packed hexagonal arrays of $\mathrm{Ni}$ nanowires: influence of geometric characteristics," Journal of Magnetism and Magnetic Materials, vol. 294, no. 2, pp. 174-181, 2005.

[19] K. Ounadjela, R. Ferré, L. Louail, et al., "Magnetization rever -sal in cobalt and nickel electrodeposited nanowires," Journal of Applied Physics, vol. 81, p. 5455, 1997.

[20] F. Li, T. Wang, L. Ren, and J. Sun, "Structure and magnetic properties of Co nanowires in self-assembled arrays," Journal of Physics Condensed Matter, vol. 16, no. 45, pp. 8053-8060, 2004.

[21] A. H. Morrish, The Phyciples of Magnetism, Wiley, New York, NY, USA, 1965.

[22] M. Darques, A. Encinas, L. Vila, and L. Piraux, "Controlled changes in the microstructure and magnetic anisotropy in arrays of electrodeposited Co nanowires induced by the solution pH," Journal of Physics D, vol. 37, no. 10, pp. 14111416, 2004. 

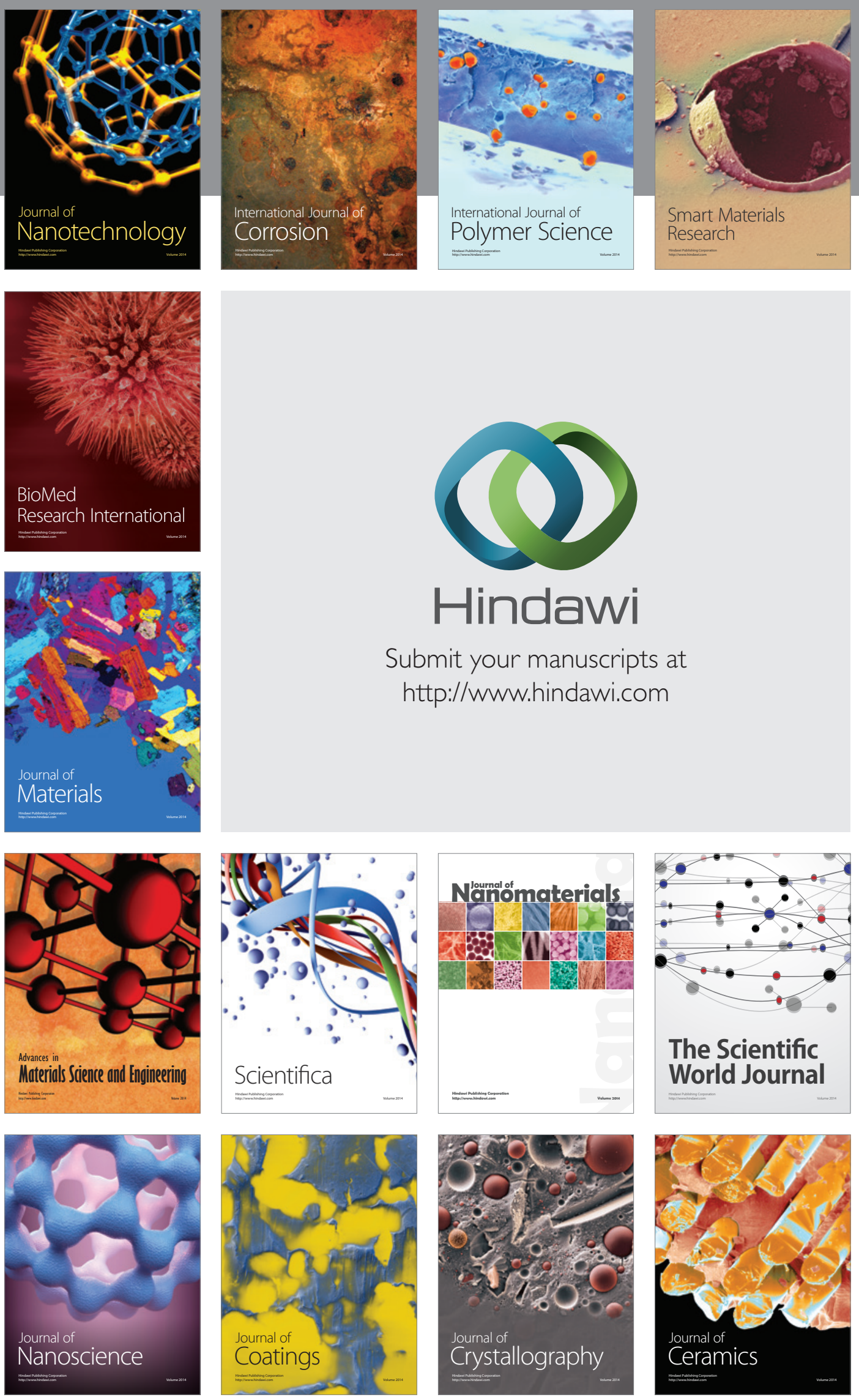

The Scientific World Journal

Submit your manuscripts at

http://www.hindawi.com

\section{World Journal}

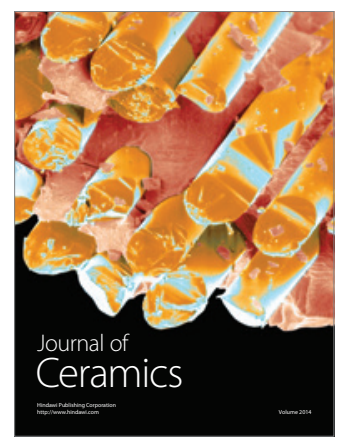

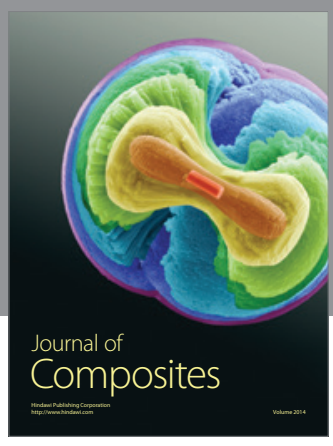
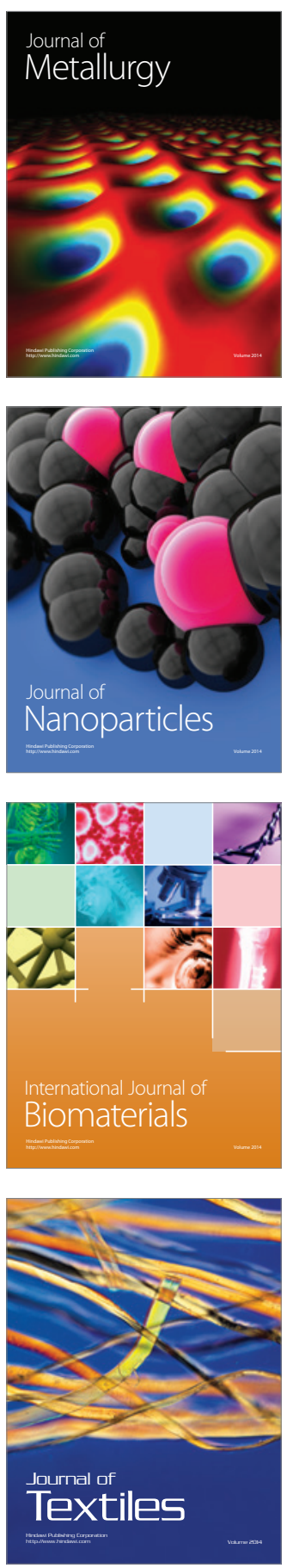